\title{
A ATENÇÃO FARMACÊUTICA NAS FARMÁCIAS COMUNITÁRIAS \\ DO MUNICÍPIO DE CAMPINA GRANDE DO SUL
}

\author{
PHARMACEUTICAL CARE IN COMMUNITY PHARMACIES \\ OF THE CITY OF CAMPINA GRANDE DO SUL
}

\begin{abstract}
BARETA, G. M. S. ${ }^{\text {** }}$
'Professora da disciplina de Deontologia e Legislação Farmacêutica do Departamento de Medicina Forense e Psiquiatria, Universidade Federal do Paraná(UFPR), Curitiba- PR.

**Correspondência: Rua Pref. Lothário Meissner, 3400, CEP 80210-170, Jardim Botânico, Curitiba, PR, gracce@subsede.ufpr.br
\end{abstract}

\section{RESUMO}

Este trabalho compõe um estudo sobre as farmácias comunitárias do Município de Campina Grande do Sul, localizada no Estado do Paraná, Brasil, com o objetivo de compreender como o conjunto de seus agentes 3/4farmacêuticos, proprietários, balconistas, farmacêuticos-proprietários e proprietários-balconistas $3 / 4$ percebem suas práticas relacionadas ao atendimento ao paciente, na perspectiva da atenção farmacêutica. A atenção farmacêutica constitui uma prática profissional farmacêutica, voltada a intervir na farmacoterapia do paciente, além da promoção e prevenção da saúde, contribuindo com o uso racional do medicamento. Dentro de uma abordagem qualitativa, discorre sobre o conceito, os fatores e relações que permeiam estas farmácias, contrapondo os dados empíricos coletados, aos dados teóricos sobre a atenção farmacêutica, originados de revisão bibliográfica. A coleta de dados realizou-se através de entrevistas semiestruturadas e observação direta, no período que compreendeu os meses de julho e agosto do ano 2002 e, posteriormente, estes dados empíricos foram categorizados para sua utilização. Concluise que, nas farmácias comunitárias estudadas, existe um caminho a ser percorrido até que a atenção farmacêutica possa fazer parte da prática nestes estabelecimentos.

Palavras chave: Atenção farmacêutica; Farmácia comunitária; Farmacoterapia

\section{ABSTRACT}

This work composes a study about the community pharmacies of the city of Campina Grande do Sul, located at the state of Parana, Brazil, with the goal of understing how the set of their agentspharmacists, owners, sellers, pharmacists owners and seller owners- realize their practices related to the attention to the patient, on the perspective of pharmaceutical care. Pharmaceutical care consists in a pharmaceutical professional practice, directed to intercede on the pharmacotherapy of the patient, besides prevention and promotion of health, contributing to the rational use of medicine. Inside a qualitative approach, it discourses about the concept, the factors and relations that lead these pharmaceutical care, originated from the bibliographic revision. The data collection was carried out by means of semi-structured interviews and direct observation, during the months of July and August 2002, and later these empiric data were categorized for analysis. The conclusion is that in the community pharmacies studied, there is a way that has to be followed until the pharmaceutical care can be part of the practice in these pharmacies.

Key words: pharmaceutical care; community pharmacy; pharmacotherapy

\section{INTRODUÇÃO}

As farmácias comunitárias, neste estudo, estão caracterizadas como estabelecimentos farmacêuticos de propriedade privada, os quais atendem diretamente o paciente na dispensação de medicamentos industrializados, em suas embalagens originais, os quais não estão inseridos em hospitais, unidades de saúde ou equivalente. Estas farmácias não manipulam medicamentos e o atendimento ao paciente acontece ao nível de atenção primária à saúde', com a responsabilidade técnica, legal e privativa, de farmacêutico.

A escolha de farmácia comunitária como denominação, fundamentou-se nas argumentações de LLIMÓs(1997) que a considera importante como potencial de comunicação, uma vez que encontra-se nas fontes secundárias de informação como palavra chave para indexar a informação científica nesta área, o que permite, no mundo globalizado, uma comunicação efetiva que não se restringe a um pais ou um idioma, mas entre os vários países e idiomas, além de diferenciar este ramo da profissão farmacêutica 
dos demais. Também é possível argumentar em favor desta denominação, considerando os estudos de TÖNNIES(1995), sobre comunidades. Neste sentido, as farmácias comunitárias, por um lado, atendem a uma comunidade porque se destinam a atenção primária à saúde e, portanto direcionam-se a atender pessoas que vivem ou trabalham próximas a ela, as quais mantêm, entre si, relações de amizade pela semelhança ou a partir das condições de trabalho ou do modo de pensar. Por outro lado, mesmo quando, no conjunto, as pessoas atendidas não caracterizem uma comunidade, nas relações entre os seus agentes ${ }^{2}$ que fazem o atendimento ao paciente e os pacientes, existe algo em comum, compartilhado entre eles, a partir das necessidades e interesses que se referem à prestação de serviço inerente a estas farmácias e que dá o sentido de comunidade a estas relações.

A OMS (1986) reconhece que cabe a estas farmácias a dispensação do medicamento e, aos profissionais farmacêuticos, nestes estabelecimentos, aconselhar estes pacientes sobre o uso dos medicamentos prescritos, assessorá-los sobre medicamentos para automedicação e fornecer a outros profissionais de atenção à saúde, incluindo os médicos, informações farmacológicas. A presença e a ação do farmacêutico, nestes estabelecimentos se fundamenta no fato que o uso racional do medicamento ${ }^{3}$ requer a aplicação de um conhecimento técnicocientífico aprofundado sobre as suas características intrínsecas, pelas reações e interações adversas que podem desencadear e sobre as doenças para as quais são úteis.

No final da década de 1980, a prática do profissional farmacêutico, nestas farmácias, ganha uma nova filosofia denominada de atenção farmacêutica (HEPLER, 1988; FARRIS; SCHOPFLOCHER, 1999), voltada para a farmacoterapia, além da promoção e prevenção da saúde. A atenção farmacêutica surge como uma prática geral que inclui serviços clínicos e controle de medicamentos (HEPLER, 1988; FARRIS;SCHOPFLOCHER, 1999) que nos anos de 1990, passa a ser discutida no Brasil para ser adaptada à sua realidade.

O fato das farmácias comunitárias brasileiras não serem de propriedade exclusiva de farmacêuticos; a permissão para instalação destas farmácias sem a adoção de critérios que limitem o número de farmácias às necessidades da região; o atendimento ao paciente realizado, em grande parte, por pessoas leigas, são fatores que favorecem o mercantilismo nelas praticado, comprometendo a qualidade dos serviços prestados (OMS, 1986; ZUBIOLI, 1992; GOEL et al, 1996; ARRAIS et al, 1997).

Neste contexto, o objetivo deste estudo é compreender como o conjunto de agentes percebem suas práticas relacionadas ao atendimento do paciente, nas farmácias comunitárias do Município de Campina Grande do Sul, na perspectiva da atenção farmacêutica, enquanto prática profissional do farmacêutico que vem sendo colocada como ideal, construída pelos próprios profissionais farmacêuticos que, decorrente dos avanços científicos e tecnológicos, sentiram a necessidade de buscar novo rumo para a sua atuação profissional, direcionado à maximizar a contribuição do farmacêutico, quanto às necessidades relacionadas ao uso de medicamentos pela sociedade.

\section{METODOLOGIA}

O número reduzido de farmácias comunitárias no Município de Campina Grande do Sul, localizada no Estado do Paraná, Brasil, com 36280 habitantes, distribuídos em uma extensão de $539,861 \mathrm{~km}^{2}$ e o fácil acesso a elas motivou a escolha destas farmácias para este estudo, na medida que havia a possibilidade de estudar o universo delas, considerando que a pesquisa qualitativa a que se propõe este trabalho, pressupõe um número reduzido 
de pessoas e uma profundidade maior, pela abertura das perguntas.

Através do Conselho Regional de Farmácia do Estado do Paraná, foi possível identificar as farmácias comunitárias existentes no Município, das quais seis participaram desta pesquisa, uma vez que duas delas pertenciam a um mesmo proprietário que negou-se a participar.

Todas as farmácias comunitárias do Município estão concentradas na área de maior densidade demográfica. Considerando, para a distribuição geográfica destas farmácias, critérios como: o número de habitantes por farmácia -no Município de Campina Grande do Sul é de 4535 habitantes por farmácia ${ }^{4}$ - e que a OMS, citada por ZUBIOLI (1992, p.48) recomenda que seja de 8000-10000; a densidade demográfica; a facilidade de acesso da população a estas farmácias, podemos considerar que 4 farmácias seriam suficientes para a demanda local, existindo um excedente de 4 farmácias comunitárias, no Município.

As farmácias comunitárias estudadas não estão inseridas em drugstores e não pertencem a redes de farmácias. Em geral os proprietários possuem apenas uma farmácia, com exceção às farmácias 4 e 5 que possuem um único proprietário. 37,5\% das farmácias comunitárias do Município de Campina Grande do Sul são de propriedade de farmacêuticos, dado que se aproxima ao percentual paranaense de $36,51 \%{ }^{5}$. As categorias e o número de agentes entrevistados que atuam nestas farmácias, encontram-se na Tabela 1, a seguir:

TABELA 1 - CATEGORIAS E NÚMERO DOS AGENTES ENTREVISTADOS POR FARMÁCIA

\begin{tabular}{|c|c|c|c|c|c|c|}
\hline \multirow{3}{*}{ Farmácias } & \multicolumn{5}{|c|}{ Categorias de Agentes } & \multirow{3}{*}{$\begin{array}{l}\text { Total de } \\
\text { Agentes }\end{array}$} \\
\hline & \multicolumn{2}{|c|}{ AgentesFarmacêuticos } & \multicolumn{3}{|c|}{ AgentesLeigos } & \\
\hline & Farmacêuticos & $\begin{array}{c}\text { Farmacêuticos } \\
\text { proprietários }\end{array}$ & Proprietários & $\begin{array}{c}\text { Proprietários } \\
\text { balconistas }\end{array}$ & Balconistas & \\
\hline Farmácia 1 & 1 & 0 & 0 & 1 & 0 & 2 \\
\hline Farmácia 2 & 1 & 0 & 0 & 1 & 1 & 3 \\
\hline Farmácia 3 & 1 & 0 & (1) 1 & 0 & 2 & 4 \\
\hline Farmácia 4 & 0 & 1 & 0 & 0 & 1 & 2 \\
\hline Farmácia 5 & (2) 0 & (3) 1 & 0 & 0 & 1 & 1 \\
\hline Farmácia 6 & 0 & 1 & 0 & 0 & 1 & 2 \\
\hline $\begin{array}{l}\text { TOTAL DE } \\
\text { AGENTES }\end{array}$ & 3 & 2 & 1 & 2 & 6 & 14 \\
\hline
\end{tabular}

(1) Este proprietário é o único, entre os entrevistados, que não atua no atendimento ao paciente.

(2) No período que foram realizadas as entrevistas esta farmácia estava sem farmacêutico.

(3) Este número não foi contado nos totais de agentes que se encontram na Tabela 1, pois este também é o proprietário da Farmácia 4.

Na coleta de dados que aconteceu nos meses de julho e agosto do ano 2002, foram utilizadas as técnicas da observação direta e entrevistas do tipo semi-estruturada, aplicadas a partir de perguntas abertas aos agentes destas farmácias. As perguntas foram elaboradas a partir da necessidade de cumprir com os objetivos deste estudo. A coleta de dados foi autorizada pelos proprietários das farmácias comunitárias e demais entrevistados que antes da coleta de dados, individualmente, foram esclarecido sobre a pesquisa e concordaram em conceder a entrevista. Os esclarecimentos e posterior consentimento foram consolidados através de um termo de consentimento livre e esclarecido. As entrevistas foram agendadas de acordo com a disponibilidade de cada um dos entrevistados, foram gravadas e, posteriormente, transcritas. Os dados empíricos oriundos das entrevistas e das observações realizadas foram categorizados de acordo as questões levantadas pelos entrevistados e o 
constatado através das observações. A partir desta categorização, os dados empíricos foram articulados com os dados originados da revisão da literatura sobre atenção farmacêutica que trás os conceitos, os fatores e relações que, no curso da história contemporânea da farmácia, permearam a origem e desenvolvimento da atenção farmacêutica.

\section{RESULTADOS E DISCUSSÃO}

Para chegar a um conceito de atenção farmacêutica mais próximo da visão dos diferentes agentes que atuam nas farmácias comunitárias estudadas foi preciso fazer uso de seus depoimentos, na medida que este conceito apareceu bastante fragmentado entre eles. Este fato sugere que não existe, entre estes agentes, um consenso sobre o que seja atenção farmacêutica, embora os elementos utilizados, por eles, para defini-la, façam parte desta prática. Para eles, a atenção farmacêutica está associada a um conjunto de atitudes, palavras e procedimentos que permitam um bom atendimento ao paciente, agindo com prudência, na medida que se procura analisar a prescrição e evitar o erro. Orientando o paciente sobre o medicamento, ou orientando-o para ir ao médico. Esclarecendo dúvidas e interagindo com o prescritor. Sabendo ouvir o paciente, pois é ele que pode dar as informações necessárias para que se possa avaliar e acompanhar o tratamento, de maneira que o paciente se sinta seguro com relação ao atendimento que recebe.

Embora o discurso dos agentes das farmácias comunitárias estudadas remeta à relação entre a atenção farmacêutica e a atuação do farmacêutico, sua atuação não esta centrada no atendimento direto do paciente, como sugere o conceito de atenção farmacêutica - prática profissional em que o paciente é o principal beneficiário das ações do farmacêutico (HEPLER;STRAND, 1990; REUNIÓN DE LA OMS, 1993). As práticas observadas e o discurso dos agentes revelam que o atendimento direto do paciente é realizado mais pelos agentes leigos do que pelo farmacêutico. Neste sentido, o proprietário-balconista declara que "a atenção farmacêutica é o bioquímico quem faz e, na ausência dele, sou eu quem faço" $\left(\mathrm{P}^{6}{ }^{6}\right)$. Este fragmento de discurso, além de ratificar a atuação dos agentes leigos no atendimento ao paciente, também é contraditório às práticas no estabelecimento, na medida que observou-se que, por duas vezes, o mesmo proprietário-balconista interrompeu a entrevista que concedia para atender pacientes, ainda que na presença ociosa do farmacêutico.

Na condição em que o atendimento ao paciente é, majoritariamente, conduzido por leigos, constatou-se que o farmacêutico desempenha papéis que se diferenciam em função de situações diversas identificadas, as quais o afastam do atendimento direto ao paciente.

Uma destas situações aparece no discurso dos agentes, onde o farmacêutico supervisiona o balconista e, neste caso, existe um espaço e uma postura do farmacêutico voltados para a sua intervenção no atendimento ao paciente realizado pelo balconista. Nas palavras do próprio farmacêutico: "Na dúvida do balconista, por exemplo, na leitura da prescrição, por mais que eu não esteja no balcão, eu estou sempre por aqui... eu tenho que estar presente para atender" (F37). A fala do balconista também representa esta questão: "Acho que quem tem que fazer a atenção farmacêutica é o farmacêutico, porque é o farmacêutico que não pode deixar o balconista fazer alguma coisa errada" (B6 ${ }^{8}$ ). Nas farmácias comunitárias estudadas, o farmacêutico que ocupa a posição de supervisor está envolvido com os aspectos administrativos gerenciais da farmácia. Em seu depoimento, o farmacêutico demonstra que o seu envolvimento com as questões administrativas o afastam do atendimento direto ao paciente: "... por mais que eu esteja fazendo as coisas da 
administração, estou sempre de olho do balcão..."(F3) Ao mesmo tempo, o farmacêuticoproprietário demonstra a sua insatisfação em executar tarefas administrativas, em detrimento de suas atribuições ligadas à profissão farmacêutica: "O bom mesmo seria que minhas atividades estivessem ligadas exclusivamente à profissão farmacêutica, às necessidades do paciente, mas aqui eu não sou só farmacêutico, sou caixa, lido com a contabilidade, com serviço de banco, enfim, faço toda a parte administrativa da farmácia."(F69) Para BIRENBAUM (1982) e GOEL et al.(1996), o farmacêutico limita sua atuação às questões administrativas e gerenciais na prática em que os negócios são preponderantes e ele acaba dedicando considerável tempo para o desenvolvimento destes negócios. O negócio, nas farmácias comunitárias estudadas, onde o medicamento é tratado como mercadoria e a farmácia como um mero comércio e não como um serviço de saúde, é sinalizado através da fala do farmacêutico que ainda tem a necessidade de demonstrar que "não está na farmácia só para vender caixinhas" ( $\left.F 7^{10}\right)$.

Outra situação identificada é a do farmacêutico-proprietário, em posição hierárquica que facilitaria sua intervenção na atuação do balconista. Porém, a fala do farmacêuticoproprietário refere-se, "a venda" do medicamento e não a sua dispensação" ", o que coloca a farmácia, também, como um mero comércio. Mas, neste caso, o discurso sugere que o farmacêutico e o balconista atuam num mesmo nível, junto ao paciente: "Com relação ao atendimento do cliente, no balcão, o balconista tem a sua liberdade de atender. Ás vezes eu interfiro, mas reconheço que é uma falha minha, pois eu estou interferindo na venda dele" (F4 ${ }^{12}$ ).

Ainda foi possível identificar uma terceira situação, onde o farmacêutico aparece subjugado pelo conhecimento e experiência do proprietário-balconista. A fala do farmacêutico deixa evidente está questão: "Com certeza eu me sinto retraído pelo conhecimento e experiência do proprietário, porque é óbvio que 40 anos de prática contam mais que dois anos" (F2 ${ }^{13}$ ).

Encontramos em FOUCAULT (1994) que a identificação do problema, o delineamento de possibilidades e riscos, o exercício do poder de decisão e intervenção que pressupõe o enfoque clínico, depende do conhecimento do observador, no caso o agente que atua nas farmácias comunitárias.

Assim, considerando os avanços e a complexidade do conhecimento que envolve as doenças, os protocolos terapêutico para tratá-las e os medicamentos em si, para avaliar todos os aspectos que envolvem a adequabilidade de uma prescrição médica ou assessorar o paciente na automedicação, na perspectiva do uso racional do medicamento (OMS, 1986), é necessário o domínio, desta pessoa que atende, de um conhecimento teórico e sua aplicação à prática. Deste modo, a relação que se estabelece entre o paciente e o agente da farmácia comunitária, no momento do atendimento, é única e decisiva para o sucesso ou não do atendimento. Pressupõe-se que o farmacêutico, pela sua formação profissional, tenha o conhecimento em química, em farmacologia, em formulações de medicamentos, em leis e normas relacionadas ao cuidado, guarda e uso destes medicamentos, aliados ao conhecimento clínico, bem como o potencial para aprofundar este conhecimento para ser aplicado a sua prática que envolve o uso do medicamento e a educação dos pacientes, trazendo como vantagem o uso racional do medicamento, em benefício direto do paciente (BARRET;VERE, 1979; BIRENBAUM, 1982; FARRIS; SCHOPFLOCHER, 1999).

Mas, para BIRENBAUM(1982), a legitimidade da profissão farmacêutica se concretiza no reconhecimento do profissional farmacêutico pela sociedade, como detentor de uma base destes conhecimentos técnicos-científicos demonstrados em sua prática profissional, compatível com a ética e voltada para o bem comum. Nas farmácias comunitárias 
estudadas o fato do farmacêutico estar legalmente apto para exercer a profissão não tem sido base suficiente para a aquisição das responsabilidades que envolvem a atenção farmacêutica. O farmacêutico revela a deficiência deste conhecimento para atuar no atendimento ao paciente, quando diz: "O farmacêutico precisa do conhecimento prático sobre medicamento e sobre a parte clínica que não possui, porque tudo depende da prática e eu acho que a universidade precisa dar mais oportunidade para o acadêmico de farmácia praticar." (F2) No discurso do balconista é que está representada a legitimidade da atuação do agente, perante o paciente: "Um balconista não interfere com o paciente do outro, pois aquele paciente já tem o costume de ser tratado por aquele balconista. ...então a gente sempre trabalha respeitando o espaço de cada um. Até a questão do sigilo. Tem coisas que você conversa com o teu paciente que ele não quer conversar com o outro, mas só com você" (B4 $\left.{ }^{14}\right)$.

Neste contexto, para os agentes das farmácias comunitárias estudadas, o conhecimento necessário para atender o paciente não está centrado no farmacêutico. Este conhecimento aparece desmembrado em teoria e prática, distribuído entre o farmacêutico que detém o conhecimento teórico adquirido no processo de formação profissional e os agentes leigos, detentores do conhecimento prático. Para o balconista, "O farmacêutico tem um histórico escolar que demonstra o seu conhecimento, mas geralmente ele não tem a prática" (B2 ${ }^{15}$ ) e o farmacêutico reconhece o conhecimento do proprietário: "Ele entende da coisa, atende bem, entende bem, faz bem o diagnóstico de infecção de garganta e de bronquite" (F1). Assim fica evidente a dificuldade do farmacêutico na aplicação do conhecimento à pratica de atendimento ao paciente e a falta de conhecimento clínico, fundamentais na atenção farmacêutica.

Neste contexto, o farmacêutico aparece, ou na posição de aprendiz, representada nas falas do balconista e do farmacêutico: "Eu conheço um farmacêutico recém formado que foi aprendendo com os balconistas sobre medicamento, porque a prática é diferente." (B526), "tudo eu estou aprendendo... estou aprendendo com o proprietário e não me sinto mal, porque mais tarde eu serei beneficiado." (F2), ou intercambiando conhecimento com o balconistas:

\footnotetext{
"O balconista tem 18 anos de prática em farmácia e eu tenho 9 anos de atividade em farmácia, então a gente pode trocar conhecimento, no sentido que o balconista tem a prática do balcão, a prática do atendimento... minha contribuição aqui dentro seria um conhecimento técnico que eu posso passar para eles, noções básicas de propriedades de medicamentos que eles não têm. Eu aprendo com eles e procuro passar conhecimento para eles também." (F3)
}

No mesmo sentido, o balconista declara: "O farmacêutico tem a parte teórica, muitas vezes, não tem a prática....então a gente associa a prática do balconista com a teoria do farmacêutico" (B4). Assim, a relação agente-paciente se consolida, na medida que se legitima a ałuação do agente, isto é, na medida que existam elementos, na visão do paciente, que expliquem e justifiquem a atuação do agente neste atendimento (BERGER; LUCKMANN, 1985).

Se a atuação do farmacêutico não está legitimada, então abre-se espaço para falta de reconhecimento deste profissional, não só por parte do paciente, mas também, pelo médico prescritor e pelos agentes leigos, pela seguridade social e pelos administradores (BARRETT E VERE, 1979; BIRENBAUM, 1982; LIPOWSKI, 1996).

A falta de reconhecimento do farmacêutico aparece em seu discurso, quando coloca o problema relacionado a sua identidade social: "Eu acho que o trabalho do farmacêutico não é reconhecido pela população, até pelos médicos. O farmacêutico é considerado, por eles, um mero balconista, infelizmente" (F1). A confusão do paciente, na distinção entre o farmacêutico e os demais agentes na farmácia comunitária, particularmente com o 
balconista, estende-se também a outros profissionais provedores de saúde: "Você tem que passar para o cliente que você não é um balconista, uma vez que o farmacêutico fez um curso universitário, ou que você não é enfermeiro, porque os clientes confundem muito" (F6). No discurso do farmacêutico também está presença a justificativa para esta falta de reconhecimento: "A maior parte da população não entende quem é o farmacêutico" (F2).

Aparece no discurso dos farmacêuticos a necessidade de definir e manter sua identidade enquanto farmacêutico, na medida que consideram que o diploma de farmacêutico deve diferenciá-los dos agentes leigos. Para eles, demonstrar esta diferença, não depende apenas do diploma, mas de sua postura nas relações: "O farmacêutico fez o curso universitário e eu penso que tem que existir a diferença entre farmacêuticos e balconistas. Para que isso aconteça, temos que manter um pouquinho de pulso rígido, porque, senão, as coisas desabam" (F3).

Os farmacêuticos e farmacêuticos-proprietários que percebem um conflito na realidade das farmácias comunitárias e em suas próprias ações, frente a esta realidade, cujo perigo na maioria das vezes não é percebido pelo paciente, o revelam em seu discurso, bem como sua impotência e o desejo e a esperança de mudança:

"A minha função aqui é orientar... mas chega uma situação que não tem jeito...por mais que eu tenha orientado o paciente sobre as inconveniências do uso daquele medicamento... ele vai na outra farmácia, onde terá a indicação que deseja. Então eu indico, embora considere minha conduta anti-ética, por mais que em parte, eu acredito ter cumprido com a minha obrigação por ter orientado... eu tenho esperança que as coisas melhorem para o farmacêutico." (F1)

\section{CONCLUSÃO}

No discurso dos agentes das farmácias comunitárias estudadas se percebe uma distorção no conceito de atenção farmacêutica, não no que se refere aos elementos que devem fazer parte desta prática, uma vez que os farmacêuticos e os agentes leigos, relacionam a atenção farmacêutica à orientação ao paciente, aos resultados do tratamento. A distorção está no fato de que é o agente leigo e não o farmacêutico que realiza, predominantemente, o atendimento ao paciente.

Para realizar uma boa orientação ao paciente, de maneira que se possa obter resultados positivos no tratamento é necessário que o agente possua conhecimento suficiente para isso, porém, nas farmácias comunitárias estudas, na percepção dos agentes leigos, suas ações que são voltadas ao atendimento do paciente, estão legitimadas por este paciente, na medida que para ele, o agente leigo detém o saber prático-clínico que é fundamental para legitimar este atendimento. O próprio farmacêutico reconhece, embora em níveis diferentes, este saber do agente leigo.

Assim, o farmacêutico, mesmo que responsável técnico legal por estas farmácias, não é indispensável no atendimento direto do paciente e este fato, aliado ao número excessivo de farmácias, sendo a maioria delas de propriedade de leigos, facilita que se perpetue a perigosa lógica de mercado, onde predomina o comércio, disfarçado de serviço de saúde, uma vez que a atuação dos agentes leigos é predominante, decorrente da relação de confiança entre agentes leigos e pacientes.

A atenção farmacêutica não é uma realidade nas farmácias comunitárias estudadas e existe um caminho a ser percorrido até que possa sê-la. Neste sentido é primordial o resgate da legitimidade da ação do farmacêutico em relação ao atendimento do paciente que se 
fundamenta na sua qualificação voltada para a atenção farmacêutica e na mudança de atitude deste farmacêutico.

\author{
NOTAS \\ 'Atenção primária à saúde é a assistência sanitária essencial, baseada em métodos e tecnologias práticos, \\ cientificamente fundamentados e socialmente aceitáveis, colocada ao alcance de todos os indivíduos e famílias \\ da comunidade, mediante sua participação plena e a +um custo que a comunidade e o país possam suportar, \\ em todas e cada uma das etapas de seu desenvolvimento, com um espírito de auto-responsabilidade e \\ autodeterminação. A atenção primária forma parte integrante tanto do sistema nacional de saúde, do qual constitui \\ a função central e o núcleo principal, como do desenvolvimento social e econômico global da comunidade. \\ Representa o primeiro nível de contato dos indivíduos, da família e da comunidade com o sistema nacional de \\ saúde, levando a atenção a saúde o mais próximo possível do lugar onde residem e trabalham as pessoas e \\ constitui o primeiro elemento de um processo permanente de assistência sanitária (CONFERÊNCIA INTERNACIONAL \\ SOBRE ATENÇÃO PRIMÁRIA À SAÚDE, 1978). \\ 2 Neste estudo, são condiderados agentes, todos aqueles que executam as funções de farmacêutico, ou de \\ proprietário, ou de balconistas, ou de farmacêutico-proprietário, ou de proprietário-balconista. \\ 3 "Uso racional do medicmanto é o processo que compreende a prescrição apropriada; a disponibilidade oportuna \\ e a preços acessíveis; a dispensação em condições adequadas; e o consumo das doses indicadas, nos intervalos \\ definidos e no período de tempo indicado de medicamentos eficazes, seguros e de qualidade." (BRASIL, 1998) \\ ${ }^{4}$ Dado calculado a partir dos dados fornecidos pelo CRF-PR-2003 e IBGE-Censo Demográfico- 2000. \\ ${ }^{5}$ Dado calculado a partir dos dados fornecidos pelo CRF-PR-2003. \\ ${ }^{6}$ P1: proprietário da farmácia 1, com 30 anos de atividade em farmácia comunitária. \\ ${ }^{7}$ F3: farmacêutico da farmácia 3, com 9 anos de atividade em farmácia comunitária. \\ 8 B6: balconista da farmácia 6, com 5 anos de atividade em farmácia comunitária. \\ 9 F6: farmacêutico da farmácia 6, com 7 anos de atividade em farmácia comunitária. \\ $10 \mathrm{Fl}$ : farmacêutico da farmácia 1, com 4 anos de atividade em farmácia comunitária. \\ 11 Dispensação de medicamento: ato profissional do farmacêutico (BRASIL, 1998). \\ 12 F4: farmacêutico da farmácia 4, com 3 anos de atividade em farmácia comunitária. \\ ${ }^{13}$ F2: farmacêutico da farmácia 2, com 2 anos de atividade em farmácia comunitária. \\ ${ }^{14}$ B4: balconista da farmácia 4, com 20 anos de atividade em farmácia comunitária. \\ ${ }^{15}$ B2: balconista da farmácia 2, com 10 anos de atividade em farmácia comunitária. \\ ${ }^{16}$ B5: balconista da farmácia 5, com 10 anos de atividade em farmácia comunitária.
}

\title{
REFERÊNCIAS
}

ARRAIS, P.S. et al. Perfil da automedicação no Brasil. Revista de Saúde Pública, São Paulo, v. 31, n. 1, p.71-77, 1977. BARRETT, C. W.; VERE, D. W. The interface between pharmacy and medicine. Journal of Clinical Pharmacy, [S.I.], V. 4, p. 159-165, 1979.

BERGER, P.L.; LUCKMANN, T. A construção social da realidade. 14 ed. Petrópolis: Vozes, 1985.

BIRENBAUM, A. Reprofessionalization in pharmacy. Social Science \& Medicine, [S.I.], v. 16, n. 8, p.871-878, 1982. BRASIL. Ministério da Saúde. Gabinete do Ministro. Portaria n. 3916 de 30 de outubro de 1998. Aprova a política nacional de medicamentos. Disponível em: http://www.anvisa.gov.br/legis/portarias/391698htm. Acesso em 13 fev. 2003.

CONFERÊNCIA INTERNACIONAL SOBRE A ATENÇÃO PRIMÁRIA À SAÚDE, 1978, Alma Ata. Declaração de Alma-Ata. Disponível em: http://whqlibdoc.who.int/publications/9243541358.pdf. Acesso em 25 mar. 2002.

FARRIS, K. B.; SCHOPFLOCHER, D.P. Between intentention and behavior: an application of community pharmacists' assessment of pharmaceutical care. Social Science \& Medicine, [S.I.], v. 49, n. 1, p. 55-66, 1999.

FOUCAULT, M. O nascimento da clínica. 4 ed. Rio de Janeiro: Forense Universitária, 1994.

GOEL, P. et al. Retail pharmacies in developing countries: a behavior and intervention framework. Social Science \& Medicine, [S.I.], v. 42, n. 8, p. 1155-1161, 1996.

HEPLER, C.D. Unresolved issues in the future of pharmacy. American Journal of Hospital Pharmacy, [S.I.], v. 45, p. 1071-1081, may. 1988.

HEPLER, C. D.; STRAND, L.M. Opportunities and responsabilities in pharmaceutical care. American Journal of Hospital Pharmacy, [S.I.], v. 47, p. 533-543, mar. 1990.

LIMMÓS, F. Concepto de farmacia comunitaria: semántica y pragmática. Farmacia Professional, [S.I.], p. 44-46, dec. 1997. LIPOWSKI, E.E. Evaluating the outcomes of pharmaceutical care. Journal of the American Pharmaceutical Association, [S.I.], v. NS36, n. 12, p. 726-736, dec. 1996.

OMS. Uso racional de los medicamentos: informe de la conferencia de expertos. Ginebra, 1986.

REUNIÓN DE LA OMS, 1993, Tókio. Informe: el papel del farmaceutico en el sistema de atención de salud. [S.I.]: OPS, 1993. $13 \mathrm{p}$

TÖNNIES, F. Livro I: determinação geral dos conceitos principais. In: MIRANDA, O. de(Org.). Para ler Ferdinand Tönnies. São Paulo: EDUSP, 1995. p. 231-172.

ZUBIOLI, A. Profissão farmacêutico: e agora? Curitiba: Lovise, 1992. 